\title{
Bifurcations of resonant double homoclinic loops for higher dimensional systems
}

\author{
Yinlai Jin ${ }^{a, *}$, Han Xü ${ }^{a}$ Yuerang Gao ${ }^{a, b}$, Xue Zhao ${ }^{a, b}$, Dandan Xie \\ ${ }^{a}$ School of Science, Linyi University, Linyi, Shandong, 276005, P. R. China. \\ ${ }^{b}$ School of Mathematical Sciences, Shandong Normal University, Jinan, 250014, P. R. China.
}

\begin{abstract}
In this work, we study the bifurcation problems of double homoclinic loops with resonant condition for higher dimensional systems. The Poincaré maps are constructed by using the foundational solutions of the linear variational systems as the local coordinate systems in the small tubular neighborhoods of the homoclinic orbits. We obtain the existence, number and existence regions of the small homoclinic loops, small periodic orbits, and the large homoclinic loops, large periodic orbits, respectively. Moreover, the complete bifurcation diagrams are given. (C)2016 All rights reserved.
\end{abstract}

Keywords: Double homoclinic loops, resonance, bifurcation, higher dimensional system.

2010 MSC: 34C23, 34C37, 37C29.

\section{Introduction and Hypotheses}

In the study of the complex dynamic behaviors of high-dimensional nonlinear dynamical systems, the bifurcation problems of homoclinic orbits and heteroclinic loops have been becoming an important research field. By using the traditional Poincaré map construction method, [1, 8, 11] discussed the bifurcations of non-degenerated homoclinic loops. In [13], Zhu discussed the bifurcation problems of non-degenerated homoclinic loop by using the generalized Floquet theory. In [3, 4, 5, 6], by using the foundational solutions of the linear variational systems of the unperturbed systems along the homoclinic orbits as the local coordinate systems to construct the Poincaré maps, the authors studied the bifurcations and stability of homoclinic loops for higher dimensional systems. In [2, 10, the stability of double homoclinic loops was studied. In [7, 12], Lu and Zhang studied the double homoclinic loops bifurcations under the non-resonant condition. In this paper, we study the bifurcations of double homoclinic loops under the resonant condition for higher

\footnotetext{
*Corresponding author

Email addresses: jinyinlai@sina.com (Yinlai Jin), xuhan@lyu.edu.cn (Han Xu), yueranggao@sina.com (Yuerang Gao), xzhaomath@163.com (Xue Zhao), xiedandan01@sina.com (Dandan Xie)
} 
dimensional systems. In this case, we get the complete bifurcation diagrams. Specially, we obtain the more complex bifurcation phenomena than that of [12].

Suppose the following $C^{r}$ system

$$
\dot{z}=f(z),
$$

where $r \geq 5, z \in \mathbf{R}^{m+n}$, satisfies the following hypotheses.

(H1) (Hyperbolicity) $z=0$ is the hyperbolic equilibrium of system (1.1), the stable manifold $W_{0}^{s}$ and the unstable manifold $W_{0}^{u}$ of $z=0$ are $m$-dimensional and $n$-dimensional respectively. $\lambda_{1}$ and $-\rho_{1}$ are simple eigenvalues of $D f(0)$, such that any other eigenvalue $\sigma$ of $D f(0)$ satisfies either $\operatorname{Re} \sigma<-\rho_{0}<$ $-\rho_{1}<0$ or $\operatorname{Re} \sigma>\lambda_{0}>\lambda_{1}>0$, where $\lambda_{0}$ and $\rho_{0}$ are some positive numbers.

(H2) (Non-degeneration) System (1.1) has a double homoclinic loops $\Gamma=\Gamma_{1} \cup \Gamma_{2}, \Gamma_{i}=\left\{z=r_{i}(t)\right.$ : $\left.t \in \mathbf{R}, r_{i}( \pm \infty)=0\right\}, i=1,2$. For any $P \in \Gamma, \operatorname{codim}\left(T_{P} W_{0}^{u}+T_{P} W_{0}^{s}\right)=1$, where, $T_{P} W_{0}^{s}$ and $T_{P} W_{0}^{u}$ are the tangent spaces of $W_{0}^{s}$ and $W_{0}^{u}$ at $P$ respectively.

(H3) (Strong inclination) $\lim _{t \rightarrow+\infty}\left(T_{r_{i}(t)} W_{0}^{s}+T_{r_{i}(t)} W_{0}^{u}\right)=T_{0} W_{0}^{s} \oplus T_{0} W_{0}^{u u}, \lim _{t \rightarrow-\infty}\left(T_{r_{i}(t)} W_{0}^{s}+T_{r_{i}(t)} W_{0}^{u}\right)=$ $T_{0} W_{0}^{s s} \oplus T_{0} W_{0}^{u}$, where $i=1,2, W_{0}^{s s}$ and $W_{0}^{u u}$ are the strong stable manifold and the strong unstable manifold of $z=0$ respectively, $T_{0} W_{0}^{s s}$ is the generalized eigenspace corresponding to those eigenvalues with smaller real part than $-\rho_{0}, T_{0} W_{0}^{u u}$ is the generalized eigenspace corresponding to those eigenvalues with larger real part than $\lambda_{0}$. Let $e_{i}^{ \pm}=\lim _{t \rightarrow \mp \infty} \dot{r}_{i}(t) /\left|\dot{r}_{i}(t)\right|, e_{i}^{+} \in T_{0} W_{0}^{u}$ and $e_{i}^{-} \in T_{0} W_{0}^{s}$ are the unit eigenvectors corresponding to $\lambda_{1}$ and $-\rho_{1}$ respectively. $e_{1}^{+}=-e_{2}^{+}, e_{1}^{-}=-e_{2}^{-} \cdot \operatorname{span}\left(T_{0} W_{0}^{u u}\right.$, $\left.e_{i}^{+}\right)=T_{0} W_{0}^{u}, \operatorname{span}\left(T_{0} W_{0}^{s s}, e_{i}^{-}\right)=T_{0} W_{0}^{s}$.

(H4) (Resonance condition) $\rho_{1}=\lambda_{1}$.

Now, we consider the bifurcation problems of the following $C^{r}$ system

$$
\dot{z}=f(z)+g(z, \mu)
$$

where $\mu \in \mathbf{R}^{l}, l \geq 3,0 \leq|\mu| \ll 1, g(0, \mu)=g(z, 0)=0$.

\section{Local coordinate systems}

Suppose that (H1) $-(\mathbf{H 3})$ are established. Then, for $|\mu| \ll 1$, in the small enough neighborhood $U$ of $z=0$, we introduce a $C^{r}$ transformation such that system $(1.2)$ has the following form

$$
\left\{\begin{array}{l}
\dot{x}=\left[\lambda_{1}(\mu)+\cdots \cdots\right] x+u[O(y)+O(v)], \\
\dot{y}=\left[-\rho_{1}(\mu)+\cdots \cdots\right] y+v[O(x)+O(u)], \\
\dot{u}=\left[B_{1}(\mu)+\cdots \cdots\right] u+x[O(x)+O(y)+O(v)], \\
\dot{v}=\left[-B_{2}(\mu)+\cdots \cdots\right] v+y[O(x)+O(y)+O(u)],
\end{array}\right.
$$

where $z=\left(x, y, u^{*}, v^{*}\right)^{*}, x \in R^{1}, y \in R^{1}, u \in \mathbf{R}^{n-1}, v \in \mathbf{R}^{m-1}, *$ means transposition, $\lambda_{1}(0)=\rho_{1}(0)$, $\operatorname{Re} \sigma\left(B_{1}(\mu)\right)>\lambda_{0}, \operatorname{Re} \sigma\left(-B_{2}(\mu)\right)<-\rho_{0}$. Moreover, the unstable manifold, stable manifold, strong unstable manifold, strong stable manifold and local homoclinic orbits have the following forms, respectively

$$
\begin{array}{ll}
W_{l o c}^{u}=\{y=0, v=0\}, & W_{l o c}^{s}=\{x=0, u=0,\}, \\
W_{l o c}^{u u}=\{x=0, y=0, v=0\}, & W_{l o c}^{s s}=\{x=0, u=0, y=0\}, \\
\Gamma_{i} \cap W_{l o c}^{u}=\left\{y=0, v=0, u=u_{i}(x)\right\}, & \Gamma_{i} \cap W_{l o c}^{s}=\left\{x=0, u=0, v=v_{i}(y)\right\},
\end{array}
$$

where $i=1,2, u_{i}(0)=\dot{u}_{i}(0)=0, v_{i}(0)=\dot{v}_{i}(0)=0$.

Denote $r_{i}(t)=\left(r_{i}^{x}(t), r_{i}^{y}(t),\left(r_{i}^{u}(t)\right)^{*},\left(r_{i}^{v}(t)\right)^{*}\right)^{*}, i=1,2$. Suppose that $r_{1}\left(-T_{1}\right)=\left(\delta, 0, \delta_{1, u}^{*}, 0^{*}\right)^{*}, r_{1}\left(T_{1}\right)=$ $\left(0, \delta, 0^{*}, \delta_{1, v}^{*}\right)^{*}, r_{2}\left(-T_{2}\right)=\left(-\delta, 0, \delta_{2, u}^{*}, 0^{*}\right)^{*}, r_{2}\left(T_{2}\right)=\left(0,-\delta, 0^{*}, \delta_{2, v}^{*}\right)^{*}$, where, $T_{i}>0, i=1,2, \delta$ is small 
enough, such that $\{(x, y, u, v):|x|,|y|,|u|,|v|<2 \delta\} \subset U$. Obviously, we have $\left|\delta_{i, u}\right|=O\left(\delta^{\omega_{1}}\right),\left|\delta_{i, v}\right|=O\left(\delta^{\omega_{2}}\right)$, $\omega_{1}=\operatorname{Re} \frac{\sigma\left(B_{1}(\mu)\right)}{\lambda_{1}(\mu)}>1, \omega_{2}=\operatorname{Re} \frac{\sigma\left(B_{2}(\mu)\right)}{\rho_{1}(\mu)}>1$.

Consider the linear system

$$
\dot{z}=\left(D f\left(r_{i}(t)\right)\right) z .
$$

Similar to [3, 5, 6, 7, 12], system (2.2) has a fundamental solution matrix $Z_{i}(t)=\left(z_{i}^{1}(t), z_{i}^{2}(t), z_{i}^{3}(t), z_{i}^{4}(t)\right)$ satisfying

$$
\begin{aligned}
z_{i}^{1}(t) & \in\left(T_{r_{i}(t)} W^{s}\right)^{c} \cap\left(T_{r_{i}(t)} W^{u}\right)^{c} \\
z_{i}^{2}(t) & =(-1)^{i} \dot{r}_{i}(t) /\left|\dot{r}_{i}^{y}\left(T_{i}\right)\right| \in T_{r_{i}(t)} W^{s} \cap T_{r_{i}(t)} W^{u}, \\
z_{i}^{3}(t) & =\left(z_{i}^{3,1}(t), \cdots, z_{i}^{3, n-1}(t)\right) \in\left(T_{r_{i}(t)} W^{s}\right)^{c} \cap\left(T_{r_{i}(t)} W^{u}\right)=T_{r_{i}(t)} W^{u u}, \\
z_{i}^{4}(t) & =\left(z_{i}^{4,1}(t), \cdots, z_{i}^{4, m-1}(t)\right) \in\left(T_{r_{i}(t)} W^{s}\right) \cap\left(T_{r_{i}(t)} W^{u}\right)^{c}=T_{r_{i}(t)} W^{s s}
\end{aligned}
$$

and

$$
Z_{i}\left(T_{i}\right)=\left(\begin{array}{llll}
1 & 0 & w_{i}^{31} & 0 \\
0 & 1 & w_{i}^{32} & 0 \\
0 & 0 & w_{i}^{33} & 0 \\
w_{i}^{14}, & w_{i}^{24} & w_{i}^{34} & I
\end{array}\right), \quad Z_{i}\left(-T_{i}\right)=\left(\begin{array}{llll}
w_{i}^{11} & w_{i}^{21} & 0 & w_{i}^{41} \\
w_{i}^{12} & 0 & 0 & w_{i}^{42} \\
w_{i}^{13} & w_{i}^{23} & I & w_{i}^{43} \\
0, & 0 & 0 & w_{i}^{44}
\end{array}\right),
$$

where $i=1,2, w_{i}^{21}<0, w_{i}^{12} \neq 0, \operatorname{det} w_{i}^{33} \neq 0$, det $w_{i}^{44} \neq 0$, and $\left|w_{i}^{1 j}\left(w_{i}^{12}\right)^{-1}\right| \ll 1, j \neq 2 ;\left|w_{i}^{2 j}\left(w_{i}^{21}\right)^{-1}\right| \ll 1$, $j=3,4 ;\left|w_{i}^{3 j}\left(w_{i}^{33}\right)^{-1}\right| \ll 1, j \neq 3 ;\left|w_{i}^{4 j}\left(w_{i}^{44}\right)^{-1}\right| \ll 1, j \neq 4$.

Denote $\Phi_{i}(t)=\left(\phi_{i}^{1}(t), \phi_{i}^{2}(t), \phi_{i}^{3}(t), \phi_{i}^{4}(t)\right)=\left(Z_{i}^{-1}(t)\right)^{*}, i=1,2$, so, $\Phi_{i}(t)$ is a fundamental solution matrix of the adjoint system $\dot{\phi}=-\left(D f\left(r_{i}(t)\right)\right)^{*} \phi$ of $[2.2)$, and $\phi_{i}^{1}(t) \in\left(T_{r_{i}(t)} W^{s}\right)^{c} \cap\left(T_{r_{i}(t)} W^{u}\right)^{c}$ is bounded and tends to zero exponentially as $t \rightarrow \pm \infty[4,7,9,12,13$.

We select $z_{i}^{1}(t), z_{i}^{2}(t), z_{i}^{3}(t), z_{i}^{4}(t)$ as the local coordinate systems along $\Gamma_{i}, i=1,2$.

Let $\Delta_{i}=w_{i}^{12} /\left|w_{i}^{12}\right|, i=1,2$. We say that $\Gamma_{i}$ is non-twisted as $\Delta_{i}=1$, and twisted as $\Delta_{i}=-1$. In this paper, we consider the case $\Delta_{1}=\Delta_{2}=1$.

\section{Poincaré Maps and the bifurcation equations}

Denote $h_{i}(t)=r_{i}(t)+Z_{i}(t) N_{i}(t), N_{i}(t)=\left(n_{i}^{1}, 0,\left(n_{i}^{3}\right)^{*},\left(n_{i}^{4}\right)^{*}\right)^{*}, i=1,2$, let $S_{i}^{-}=\left\{z=h_{i}\left(-T_{i}\right)\right.$ : $|x|,|y|,|u|,|v|<2 \delta\} \subset U, S_{i}^{+}=\left\{z=h_{i}\left(T_{i}\right):|x|,|y|,|u|,|v|<2 \delta\right\} \subset U$ be the cross sections of $\Gamma_{i}$ at $t=-T_{i}$ and $t=T_{i}$, respectively.

Now, we set up Poincaré maps. (Figure 1)

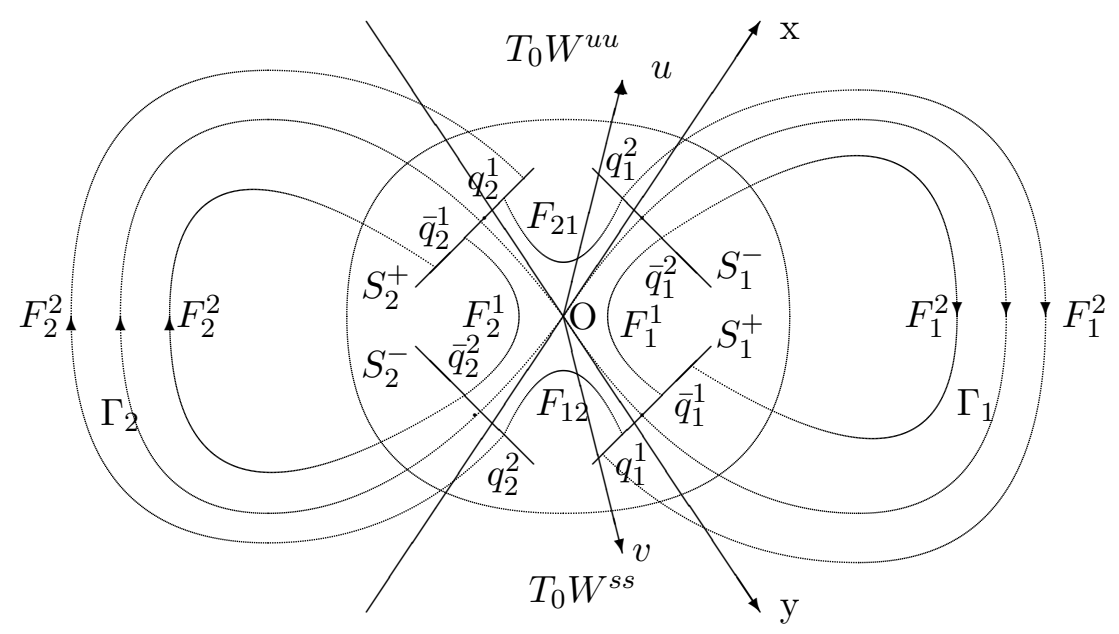

Figure 1 
In $U$, denote $F_{21}: S_{2}^{+} \rightarrow S_{1}^{-}, F_{21}\left(q_{2}^{2 j+1}\right)=q_{1}^{2 j+2} ; F_{12}: S_{1}^{+} \rightarrow S_{2}^{-}, F_{12}\left(q_{1}^{2 j+1}\right)=q_{2}^{2 j+2} ; F_{1}^{1}: S_{1}^{+} \rightarrow S_{1}^{-}$, $F_{1}^{1}\left(\bar{q}_{1}^{2 j+1}\right)=\bar{q}_{1}^{2 j+2} ; F_{2}^{1}: S_{2}^{+} \rightarrow S_{2}^{-}, F_{2}^{1}\left(\bar{q}_{2}^{2 j+1}\right)=\bar{q}_{2}^{2 j+2}$; where $i=1,2, j=0,1, \cdots$ In the tubular neighborhood of $\Gamma_{i}$, let $F_{i}^{2}$ be the map from $S_{i}^{-}$to $S_{i}^{+}, F_{i}^{2}\left(q_{i}^{2 j}\right)=q_{i}^{2 j+1}, F_{i}^{2}\left(\bar{q}_{i}^{2 j}\right)=\bar{q}_{i}^{2 j+1}$, where $i=1,2$, $j=1,2, \cdots$.

At first, we set up the relationship between the Cartesian coordinates and the normal coordinates of the points in the neighborhood of homoclinic loop. Let

$$
\begin{gathered}
q_{i}^{2 j+2}\left(x_{i}^{2 j+2}, y_{i}^{2 j+2},\left(u_{i}^{2 j+2}\right)^{*},\left(v_{i}^{2 j+2}\right)^{*}\right)^{*}=r_{i}\left(-T_{i}\right)+Z_{i}\left(-T_{i}\right) N_{i}^{2 j+2}, \\
\bar{q}_{i}^{2 j+2}\left(\bar{x}_{i}^{2 j+2}, \bar{y}_{i}^{2 j+2},\left(\bar{u}_{i}^{2 j+2}\right)^{*},\left(\bar{v}_{i}^{2 j+2}\right)^{*}\right)^{*}=r_{i}\left(-T_{i}\right)+Z_{i}\left(-T_{i}\right) \bar{N}_{i}^{2 j+2}, \\
q_{i}^{2 j+1}\left(x_{i}^{2 j+1}, y_{i}^{2 j+1},\left(u_{i}^{2 j+1}\right)^{*},\left(v_{i}^{2 j+1}\right)^{*}\right)^{*}=r_{i}\left(T_{i}\right)+Z\left(T_{i}\right) N_{i}^{2 j+1}, \\
\bar{q}_{i}^{2 j+1}\left(\bar{x}_{i}^{2 j+1}, \bar{y}_{i}^{2 j+1},\left(\bar{u}_{i}^{2 j+1}\right)^{*},\left(\bar{v}_{i}^{2 j+1}\right)^{*}\right)^{*}=r_{i}\left(T_{i}\right)+Z\left(T_{i}\right) \bar{N}_{i}^{2 j+1}, \\
N_{i}^{2 j+2}=\left(n_{i}^{2 j+2,1}, 0,\left(n_{i}^{2 j+2,3}\right)^{*},\left(n_{i}^{2 j+2,4}\right)^{*}\right)^{*}, \\
N_{i}^{2 j+1}=\left(n_{i}^{2 j+1,1}, 0,\left(n_{i}^{2 j+1,3}\right)^{*},\left(n_{i}^{2 j+1,4}\right)^{*}\right)^{*}, \\
\bar{N}_{i}^{2 j+2}=\left(\bar{n}_{i}^{2 j+2,1}, 0,\left(\bar{n}_{i}^{2 j+2,3}\right)^{*},\left(\bar{n}_{i}^{2 j+2,4}\right)^{*}\right)^{*}, \\
\bar{N}_{i}^{2 j+1}=\left(\bar{n}_{i}^{2 j+1,1}, 0,\left(\bar{n}_{i}^{2 j+1,3}\right)^{*},\left(\bar{n}_{i}^{2 j+1,4}\right)^{*}\right)^{*} .
\end{gathered}
$$

By $Z_{i}^{-1}\left(T_{i}\right), Z_{i}^{-1}\left(-T_{i}\right)$, we get

$$
y_{1}^{2 j+1} \approx \delta, x_{1}^{2 j+2} \approx \delta, y_{2}^{2 j+1} \approx-\delta, x_{2}^{2 j+2} \approx-\delta
$$

and

$$
\begin{aligned}
& \left\{\begin{array}{l}
n_{i}^{2 j+2,1}=\left(w_{i}^{12}\right)^{-1}\left[y_{i}^{2 j+2}-w_{i}^{42}\left(w_{i}^{44}\right)^{-1} v_{i}^{2 j+2}\right], \\
n_{i}^{2 j+2,3}=u_{i}^{2 j+2}-\delta_{i u}+b_{i}\left(w_{i}^{12}\right)^{-1} y_{i}^{2 j+2}+a_{i}\left(w_{i}^{44}\right)^{-1} v_{i}^{2 j+2}, \\
n_{i}^{2 j+2,4}=\left(w_{i}^{44}\right)^{-1} v_{i}^{2 j+2}
\end{array}\right. \\
& \left\{\begin{array}{l}
n_{i}^{2 j+1,1}=x_{i}^{2 j+1}-w_{i}^{31}\left(w_{i}^{33}\right)^{-1} u_{i}^{2 j+1}, \\
n_{i}^{2 j+1,3}=\left(w_{i}^{33}\right)^{-1} u_{i}^{2 j+1} \\
n_{i}^{2 j+1,4}=-w_{i}^{14} x_{i}^{2 j+1}+c_{i}\left(w_{i}^{33}\right)^{-1} u_{i}^{2 j+1}+v_{i}^{2 j+1}-\delta_{i v},
\end{array}\right.
\end{aligned}
$$

where, $b_{i}=w_{i}^{11} w_{i}^{23}\left(w_{i}^{21}\right)^{-1}-w_{i}^{13}, a_{i}=-w_{i}^{43}+w_{i}^{13}\left(w_{i}^{12}\right)^{-1} w_{i}^{42}-w_{i}^{23}\left(w_{i}^{21}\right)^{-1}\left[-w_{i}^{41}+w_{i}^{11}\left(w_{i}^{12}\right)^{-1} w_{i}^{42}\right]$, $c_{i}=\left(w_{i}^{14} w_{i}^{31}+w_{i}^{24} w_{i}^{32}-w_{i}^{34}\right)$.

As well, the relationship between the two kinds coordinates of $\bar{q}_{i}^{2 j+2}, \bar{q}_{i}^{2 j+1}$ also satisfies (3.1), (3.2) and (3.3).

Now, we consider the map $F_{i}^{2}$. Substituting transformation $z=h_{i}(t)$ into $(1.2)$, and using $\dot{r}_{i}(t)=$ $f\left(r_{i}(t)\right), \dot{Z}_{i}(t)=D f\left(r_{i}(t)\right) Z_{i}(t)$, we get

$$
Z_{i}(t)\left(\dot{n_{i}^{1}}, 0,\left(\dot{n}_{i}^{3}\right)^{*},\left(\dot{n_{i}^{4}}\right)^{*}\right)^{*}=g_{\mu}\left(r_{i}(t), 0\right) \mu+\text { h.o.t. . }
$$

Multiplying the both sides of the above equation by $\Phi_{i}^{*}(t)$ and using $\Phi_{i}^{*}(t) Z_{i}(t)=I$, we have

$$
\left(\dot{n_{i}^{1}}, 0,\left(\dot{n_{i}^{3}}\right)^{*},\left(\dot{n_{i}^{4}}\right)^{*}\right)^{*}=\Phi_{i}^{*}(t) g_{\mu}\left(r_{i}(t), 0\right) \mu+\text { h.o.t. . }
$$

Integrating it, we have $F_{i}^{2}$ defined by the following

$$
\left\{\begin{array}{l}
n_{i}^{2 j+3, k}=n_{i}^{2 j+2, k}+M_{i}^{k} \mu+\text { h.o.t. }, \quad k=1,3,4, \\
\bar{n}_{i}^{2 j+3, k}=\bar{n}_{i}^{2 j+2, k}+M_{i}^{k} \mu+\text { h.o.t. }
\end{array}\right.
$$

where, $M_{i}^{k}=\int_{-\infty}^{+\infty}\left(\phi_{i}^{k}(t)\right)^{*} g_{\mu}\left(r_{i}(t), 0\right) d t, k=1,3,4, i=1,2$.

Next, we consider the map in $U$. For convenience, we may assume $\rho_{1}(\mu)=(1+\alpha(\mu)) \lambda_{1}(\mu)$, where, $\alpha(\mu) \in R^{1},|\alpha(\mu)| \ll 1, \alpha(0)=0$. 
Assume that $\tau_{21}$ is the flying time from $q_{2}^{1}$ to $q_{1}^{2}, \tau_{12}$ is the time from $q_{1}^{1}$ to $q_{2}^{2}, \tau_{1}$ is the time from $\bar{q}_{1}^{1}$ to $\bar{q}_{1}^{2}, \tau_{2}$ is the time from $\bar{q}_{2}^{1}$ to $\bar{q}_{2}^{2}$. Set $s_{j}=e^{-\lambda_{1}(\mu) \tau_{j}}, j=21,12,1,2$, which are called the Silnikov times. By (2.1), we have

$$
\begin{array}{ll}
x=e^{\lambda_{1}(\mu)(t-T-\tau)} x^{2}+\text { h.o.t. }, & y=e^{-(1+\alpha(\mu)) \lambda_{1}(\mu)(t-T)} y^{1}+\text { h.o.t. } \\
u=e^{B_{1}(\mu)(t-T-\tau)} u^{2}+\text { h.o.t. }, & v=e^{-B_{2}(\mu)(t-T)} v^{1}+\text { h.o.t. }
\end{array}
$$

Neglecting the higher order terms, the above formulas defined the following maps:

$$
\begin{aligned}
& F_{1}^{1}: \bar{x}_{1}^{1} \approx \delta s_{1}, \bar{y}_{1}^{2} \approx \delta s_{1}^{(1+\alpha(\mu))}, \bar{u}_{1}^{1} \approx s_{1}^{B_{1}(\mu) / \lambda_{1}(\mu)} \bar{u}_{1}^{2}, \bar{v}_{1}^{2} \approx s_{1}^{B_{2}(\mu) / \lambda_{1}(\mu)} \bar{v}_{1}^{1} . \\
& F_{2}^{1}: \bar{x}_{2}^{1} \approx-\delta s_{2}, \bar{y}_{2}^{2} \approx-\delta s_{2}^{(1+\alpha(\mu))}, \bar{u}_{2}^{1} \approx s_{2}^{B_{1}(\mu) / \lambda_{1}(\mu)} \bar{u}_{2}^{2}, \bar{v}_{2}^{2} \approx s_{2}^{B_{2}(\mu) / \lambda_{1}(\mu)} \bar{v}_{2}^{1} . \\
& F_{21}: x_{2}^{1} \approx \delta s_{21}, y_{1}^{2} \approx-\delta s_{21}^{(1+\alpha(\mu))}, u_{2}^{1} \approx s_{21}^{B_{1}(\mu) / \lambda_{1}(\mu)} u_{1}^{2}, v_{1}^{2} \approx s_{21}^{B_{2}(\mu) / \lambda_{1}(\mu)} v_{2}^{1} . \\
& F_{12}: x_{1}^{1} \approx-\delta s_{12}, y_{2}^{2} \approx \delta s_{12}^{(1+\alpha(\mu))}, u_{1}^{1} \approx s_{12}^{B_{1}(\mu) / \lambda_{1}(\mu)} u_{2}^{2}, v_{2}^{2} \approx s_{12}^{B_{2}(\mu) / \lambda_{1}(\mu)} v_{1}^{1} .
\end{aligned}
$$

At last, by (3.2)-3.4) and (3.5)-(3.8), we can get Poincaré maps as follows:

$\bar{F}_{1}=F_{1}^{2} \circ F_{1}^{1}$ is

$$
\left\{\begin{array}{l}
\bar{n}_{1}^{3,1}=\left(w_{1}^{12}\right)^{-1} \delta s_{1}^{(1+\alpha(\mu))}+M_{1}^{1} \mu+\text { h.o.t. } \\
\bar{n}_{1}^{3,3}=\bar{u}_{1}^{2}-\delta_{1 u}-b_{1}\left(w_{1}^{12}\right)^{-1} \delta s_{1}^{(1+\alpha(\mu))}+M_{1}^{3} \mu+\text { h.o.t. } \\
\bar{n}_{1}^{3,4}=\left(w_{1}^{44}\right)^{-1} s_{1}^{B_{2}(\mu) / \lambda_{1}(\mu)} \bar{v}_{1}^{1}+M_{1}^{4} \mu+\text { h.o.t. }
\end{array}\right.
$$

$\bar{F}_{2}=F_{2}^{2} \circ F_{2}^{1}$ is

$$
\left\{\begin{array}{l}
\bar{n}_{2}^{3,1}=-\left(w_{2}^{12}\right)^{-1} \delta s_{2}^{(1+\alpha(\mu))}+M_{2}^{1} \mu+\text { h.o.t. } \\
\bar{n}_{2}^{3,3}=\bar{u}_{2}^{2}-\delta_{2 u}-b_{2}\left(w_{2}^{12}\right)^{-1} \delta s_{2}^{(1+\alpha(\mu))}+M_{2}^{3} \mu+\text { h.o.t. } \\
\bar{n}_{2}^{3,4}=\left(w_{2}^{44}\right)^{-1} s_{2}^{B_{2}(\mu) / \lambda_{1}(\mu)} \bar{v}_{2}^{1}+M_{2}^{4} \mu+\text { h.o.t. }
\end{array}\right.
$$

$F_{1}=F_{1}^{2} \circ F_{21}$ is

$$
\left\{\begin{array}{l}
n_{1}^{3,1}=-\left(w_{1}^{12}\right)^{-1} \delta s_{21}^{(1+\alpha(\mu))}+M_{1}^{1} \mu+\text { h.o.t. } \\
n_{1}^{3,3}=u_{1}^{2}-\delta_{1 u}-b_{1}\left(w_{1}^{12}\right)^{-1} \delta s_{21}^{(1+\alpha(\mu))}+M_{1}^{3} \mu+\text { h.o.t. } \\
n_{1}^{3,4}=\left(w_{1}^{44}\right)^{-1} s_{21}^{B_{2}(\mu) / \lambda_{1}(\mu)} v_{2}^{1}+M_{1}^{4} \mu+\text { h.o.t. }
\end{array}\right.
$$

$F_{2}=F_{2}^{2} \circ F_{12}$ is

$$
\left\{\begin{array}{l}
n_{2}^{3,1}=\left(w_{2}^{12}\right)^{-1} \delta s_{12}^{(1+\alpha(\mu))}+M_{2}^{1} \mu+\text { h.o.t. } \\
n_{2}^{3,3}=u_{2}^{2}-\delta_{2 u}+b_{2}\left(w_{2}^{12}\right)^{-1} \delta s_{12}^{(1+\alpha(\mu))}+M_{2}^{3} \mu+\text { h.o.t. } \\
n_{2}^{3,4}=\left(w_{2}^{44}\right)^{-1} s_{12}^{B_{2}(\mu) / \lambda_{1}(\mu)} v_{1}^{1}+M_{2}^{4} \mu+\text { h.o.t. }
\end{array}\right.
$$

Meanwhile, we get the successor functions as follows:

$\bar{G}_{1}\left(s_{1}, \bar{u}_{1}^{2}, \bar{v}_{1}^{1}\right)=\left(\bar{G}_{1}^{1}, \bar{G}_{1}^{3}, \bar{G}_{1}^{4}\right)=\left(\bar{F}_{1}\left(\bar{q}_{1}^{1}\right)-\bar{q}_{1}^{1}\right)$ is

$$
\begin{aligned}
& \left\{\begin{aligned}
\bar{G}_{1}^{1}= & \delta\left[\left(w_{1}^{12}\right)^{-1} s_{1}^{(1+\alpha(\mu))}-s_{1}\right]+M_{1}^{1} \mu+\text { h.o.t. } \\
\bar{G}_{1}^{3}= & \bar{u}_{1}^{2}-\delta_{1 u}-b_{1}\left(w_{1}^{12}\right)^{-1} \delta s_{1}^{(1+\alpha(\mu))}-\left(w_{1}^{33}\right)^{-1} s_{1}^{B_{1}(\mu) / \lambda_{1}(\mu)} \bar{u}_{1}^{2} \\
& +M_{1}^{3} \mu+\text { h.o.t. } \\
\bar{G}_{1}^{4}= & -\bar{v}_{1}^{1}+\delta_{1 v}+w_{1}^{14} \delta s_{1}+\left(w_{1}^{44}\right)^{-1} s_{1}^{B_{2}(\mu) / \lambda_{1}(\mu)} \bar{v}_{1}^{1}+M_{1}^{4} \mu+\text { h.o.t. }
\end{aligned}\right. \\
& \bar{G}_{2}\left(s_{2}, \bar{u}_{2}^{2}, \bar{v}_{2}^{1}\right)=\left(\bar{G}_{2}^{1}, \bar{G}_{2}^{3}, \bar{G}_{2}^{4}\right)=\left(\bar{F}_{2}\left(\bar{q}_{2}^{1}\right)-\bar{q}_{2}^{1}\right) \text { is } \\
& \left\{\begin{aligned}
\bar{G}_{2}^{1}= & \delta\left[-\left(w_{2}^{12}\right)^{-1} s_{2}^{(1+\alpha(\mu))}+s_{2}\right]+M_{2}^{1} \mu+\text { h.o.t. } \\
\bar{G}_{2}^{3}= & \bar{u}_{2}^{2}-\delta_{2 u}-b_{2}\left(w_{2}^{12}\right)^{-1} \delta s_{2}^{(1+\alpha(\mu))}-\left(w_{2}^{33}\right)^{-1} s_{2}^{B_{1}(\mu) / \lambda_{1}(\mu)} \bar{u}_{2}^{2} \\
& +M_{2}^{3} \mu+\text { h.o.t. } \\
\bar{G}_{2}^{4}= & -\bar{v}_{2}^{1}+\delta_{2 v}-w_{2}^{14} \delta s_{2}+\left(w_{2}^{44}\right)^{-1} s_{2}^{B_{2}(\mu) / \lambda_{1}(\mu)} \bar{v}_{2}^{1}+M_{2}^{4} \mu+\text { h.o.t. }
\end{aligned}\right.
\end{aligned}
$$




$$
\begin{aligned}
& G\left(s_{12}, s_{21}, u_{1}^{2}, u_{2}^{2}, v_{1}^{1}, v_{2}^{1}\right)=\left(G_{1}^{1}, G_{1}^{3}, G_{1}^{4}, G_{2}^{1}, G_{2}^{3}, G_{2}^{4}\right)=\left(F_{1}\left(q_{2}^{1}\right)-q_{1}^{1}, F_{2}\left(q_{1}^{1}\right)-q_{2}^{1}\right) \text { is } \\
& \left\{\begin{aligned}
G_{1}^{1}= & \delta\left[-\left(w_{1}^{12}\right)^{-1} s_{21}^{(1+\alpha(\mu))}+s_{12}\right]+M_{1}^{1} \mu+\text { h.o.t. }, \\
G_{1}^{3}= & u_{1}^{2}-\delta_{1 u}-b_{1}\left(w_{1}^{12}\right)^{-1} \delta s_{21}^{(1+\alpha(\mu))}-\left(w_{1}^{33}\right)^{-1} s_{12}^{B_{1}(\mu) / \lambda_{1}(\mu)} u_{2}^{2} \\
& +M_{1}^{3} \mu+h . o . t . \\
G_{1}^{4}= & -v_{1}^{1}+\delta_{1 v}-w_{1}^{14} \delta s_{12}+\left(w_{1}^{44}\right)^{-1} s_{21}^{B_{2}(\mu) / \lambda_{1}(\mu)} v_{2}^{1}+M_{1}^{4} \mu+\text { h.o.t. }, \\
G_{2}^{1}= & \delta\left[\left(w_{2}^{12}\right)^{-1} s_{12}^{(1+\alpha(\mu))}-s_{21}\right]+M_{2}^{1} \mu+\text { h.o.t. } \\
G_{2}^{3}= & u_{2}^{2}-\delta_{2 u}+b_{2}\left(w_{2}^{12}\right)^{-1} \delta s_{12}^{(1+\alpha(\mu))}-\left(w_{2}^{33}\right)^{-1} s_{21}^{B_{1}(\mu) / \lambda_{1}(\mu)} u_{1}^{2} \\
& +M_{2}^{3} \mu+\text { h.o.t. } \\
G_{2}^{4}= & -v_{2}^{1}+\delta_{2 v}+w_{2}^{14} \delta s_{21}+\left(w_{2}^{44}\right)^{-1} s_{12}^{B_{2}(\mu) / \lambda_{1}(\mu)} v_{1}^{1}+M_{2}^{4} \mu+\text { h.o.t. . }
\end{aligned}\right.
\end{aligned}
$$

Thus, we get the three bifurcation equations as follows:

$$
\begin{aligned}
\bar{G}_{1}\left(s_{1}, \bar{u}_{1}^{2}, \bar{v}_{1}^{1}\right) & =\left(\bar{G}_{1}^{1}, \bar{G}_{1}^{3}, \bar{G}_{1}^{4}\right)=0 . \\
\bar{G}_{2}\left(s_{2}, \bar{u}_{2}^{2}, \bar{v}_{2}^{1}\right) & =\left(\bar{G}_{2}^{1}, \bar{G}_{2}^{3}, \bar{G}_{2}^{4}\right)=0 . \\
G\left(s_{12}, s_{21}, u_{1}^{2}, u_{2}^{2}, v_{1}^{1}, v_{2}^{1}\right) & =\left(G_{1}^{1}, G_{1}^{3}, G_{1}^{4}, G_{2}^{1}, G_{2}^{3}, G_{2}^{4}\right)=0 .
\end{aligned}
$$

Obviously, for system (1.2), there is an one to one correspondence between the 1-homoclinic loops and 1periodic orbits bifurcated from $\Gamma$ and the solutions of the bifurcation equations satisfy $s_{j} \geq 0, j=1,2,21,12$.

We call the 1-homoclinic loop and 1-periodic orbit bifurcated from single homoclinic loop $\Gamma_{i}$ as small homoclic loop and small period orbit, respectively; call the 1-homoclinic loop and 1-periodic orbit bifurcated from $\Gamma=\Gamma_{1} \cup \Gamma_{2}$ as large homoclic loop and large period orbit, respectively.

\section{Resonant bifurcations}

At first, we consider the bifurcations of the single homoclinic loop $\Gamma_{i}, i=1,2$.

Theorem 4.1. Suppose $(\mathbf{H 1})-(\mathbf{H} 4)$ are fulfilled, $|\mu| \ll 1, \alpha(\mu)\left(1-w_{i}^{12}\right)>0$. If $M_{i}^{1} \mu \neq 0$, then, there exist two $(l-1)$-dimensional surfaces $\Sigma_{i} \in\left\{\mu: \alpha(\mu)(-1)^{i+1} M_{1}^{1} \mu>0\right\}$, and $L_{i}$, which have the same normal vector $M_{i}^{1}$, such that

(1) System (1.2) has a unique 2-multiple 1-periodic orbit near $\Gamma_{i}$ if and only if $\mu \in \Sigma_{i}$.

(2) System (1.2) has no 1-homoclinic and 1-periodic orbit near $\Gamma_{i}$ if and only if

$$
\mu \in\left\{\alpha(\mu)>0,(-1)^{i+1} M_{i}^{1} \mu>(-1)^{i+1} \beta_{i}(\mu)\right\} \text { or } \mu \in\left\{\alpha(\mu)<0,(-1)^{i+1} M_{i}^{1} \mu<(-1)^{i+1} \beta_{i}(\mu)\right\} .
$$

(3) System 1.2 has exactly two 1-periodic orbits near $\Gamma_{i}$ if and only if

$$
\begin{aligned}
& \mu \in\left\{\alpha(\mu)>0,(-1)^{i+1} \beta_{i}^{0}(\mu)<(-1)^{i+1} M_{i}^{1} \mu<(-1)^{i+1} \beta_{i}(\mu)\right\} \\
& \mu \in\left\{\alpha(\mu)<0,(-1)^{i+1} \beta_{i}(\mu)<(-1)^{i+1} M_{i}^{1} \mu<(-1)^{i+1} \beta_{i}^{0}(\mu)\right\} .
\end{aligned}
$$

(4) System (1.2) has exactly one 1-homoclinic orbit and one 1-periodic orbit near $\Gamma_{i}$ if and only if $\mu \in L_{i}$.

(5) System (1.2) has exactly one 1-periodic orbit near $\Gamma_{i}$ if and only if

$$
\mu \in\left\{\alpha(\mu)>0,(-1)^{i+1} M_{i}^{1} \mu<(-1)^{i+1} \beta_{i}^{0}(\mu)\right\} \text { or } \mu \in\left\{\alpha(\mu)<0,(-1)^{i+1} M_{i}^{1} \mu>(-1)^{i+1} \beta_{i}^{0}(\mu)\right\} .
$$

Where, for $i=1,2, L_{i}:=\left\{\mu: M_{i}^{1} \mu=\beta_{i}^{0}(\mu)\right\}$ is a surface defined by

$$
s_{i}{ }^{1+\alpha(\mu)}=w_{i}^{12}\left(s_{i}+(-1)^{i} \delta^{-1} M_{i}^{1} \mu\right)+\text { h.o.t. , }
$$

with $s_{i}=0, \Sigma_{i}$ is a surface defined by

$$
M_{i}^{1} \mu=\beta_{i}(\mu):=(-1)^{i+1} \delta\left(w_{i}^{12}\right)^{\frac{1}{\alpha(\mu)}} \alpha(\mu)(1+\alpha(\mu))^{-1-\frac{1}{\alpha(\mu)}}+\text { h.o.t. . }
$$


Proof. For $\Gamma_{1}$, it is easy to see that, for $0 \leq s_{1},|\mu| \ll 1$, equation $\left(\bar{G}_{1}^{3}, \bar{G}_{1}^{4}\right)=0$ of $(3.16)$ always has a unique solution $\bar{u}_{1}^{2}=\bar{u}_{1}^{2}\left(s_{1}, \mu\right), \bar{v}_{1}^{1}=\bar{v}_{1}^{1}\left(s_{1}, \mu\right)$. Substituting it into $\bar{G}_{1}^{1}=0$, we get the bifurcation equation as

$$
\delta\left[\left(w_{1}^{12}\right)^{-1} s_{1}^{(1+\alpha(\mu))}-s_{1}\right]+M_{1}^{1} \mu+\text { h.o.t. }=0 .
$$

Similarly, about the bifurcation of $\Gamma_{2}$, we have the bifurcation equation as

$$
\delta\left[-\left(w_{2}^{12}\right)^{-1} s_{2}^{(1+\alpha(\mu))}+s_{2}\right]+M_{2}^{1} \mu+\text { h.o.t. }=0 .
$$

By the analysis of the existence of solutions of the equations (4.3) and 4.4 which satisfy $s_{j} \geq 0$, we get the results of the theorem. The method of the analysis is similar to that of [3], we don't state in detail here.

$\Sigma_{i}$ is called 2-multiple 1-periodic orbit bifurcation surface, $L_{i}$ is called 1-homoclinic orbit bifurcation surface. The bifurcations diagrams of Theorem 4.1 are the Figures 2, 3, 4 and 5 .

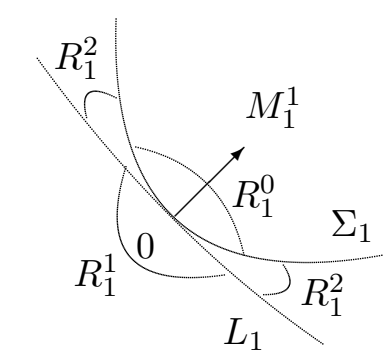

$\alpha(\mu)>0,0<w_{1}^{12}<1$

Figure 2

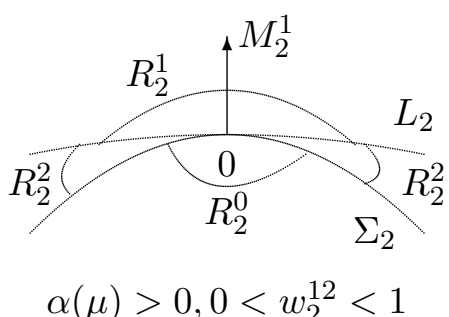

Figure 4

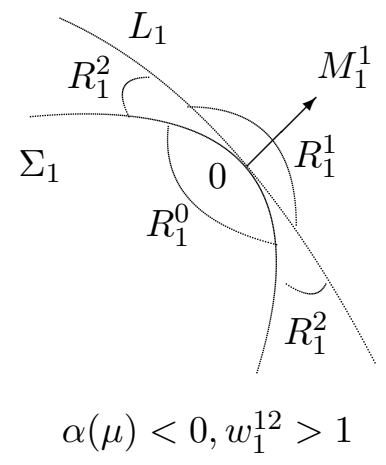

Figure 3

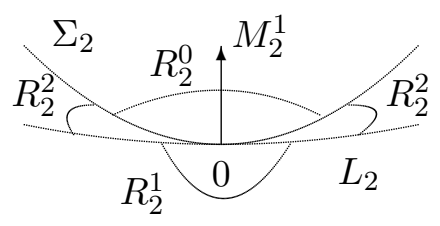

$\alpha(\mu)<0, w_{2}^{12}>1$

Figure 5

Theorem 4.2. Suppose $(\mathbf{H 1})-(\mathbf{H} 4)$ are fulfilled, $|\mu| \ll 1, \alpha(\mu)\left(1-w_{i}^{12}\right)<0$, then, we have

(1) If $(-1)^{i+1} \alpha(\mu)\left(M_{i}^{1} \mu-\beta_{i}^{0}(\mu)\right)>0$, then, system (1.2) has a unique 1-periodic orbit near $\Gamma_{i}$.

(2) If $(-1)^{i+1} \alpha(\mu)\left(M_{i}^{1} \mu-\beta_{i}^{0}(\mu)\right)<0$, then, system 1.2) has no 1-periodic orbit near $\Gamma_{i}$.

Proof. By the definition of $L_{i}$ and some simple analysis for the intersection points of the curve $Y=s_{i}{ }^{1+\alpha(\mu)}$ and the line $Y=w_{i}^{12}\left(s_{i}+(-1)^{i} \delta^{-1} M_{i}^{1} \mu\right)+$ h.o.t., we get the conclusions of this theorem.

Theorem 4.3. Suppose (H1)-(H4) are fulfilled, $|\mu| \ll 1, \alpha(\mu)=0, w_{i}^{12} \neq 1$. If $M_{i}^{1} \mu \neq 0$, then, we have the following.

(1) System 1.2 has a unique 1-periodic orbit near $\Gamma_{i}$ if and only if $\left[\left(w_{i}^{12}\right)^{-1}-1\right]^{-1}(-1)^{i+1} M_{i}^{1} \mu<0$. 
(2) System 1.2 has no 1-homoclinic orbit and 1-periodic orbit near $\Gamma_{i}$ if and only if

$$
\left[\left(w_{i}^{12}\right)^{-1}-1\right]^{-1}(-1)^{i+1} M_{i}^{1} \mu>0 .
$$

(3) System (1.2) has exactly a unique 1-homoclinic loop near $\Gamma_{i}$ if and only if $\mu \in L_{i}$, where, $L_{i}$ is defined by the following equation with $s_{i}=0$.

$$
\delta\left[\left(w_{i}^{12}\right)^{-1}-1\right] s_{i}+(-1)^{i+1} M_{i}^{1} \mu+\text { h.o.t. }=0 .
$$

Proof. If $\alpha(\mu)=0$, then, by (4.1), we only need to consider the solution of bifurcation equation (4.5).

So, if $\left(w_{i}^{12}\right)^{-1} \neq 1$, we have the unique solution $s_{i}=(-1)^{i} \delta^{-1}\left[\left(w_{i}^{12}\right)^{-1}-1\right]^{-1} M_{i}^{1} \mu+$ h.o.t..

For the bifurcations diagrams of Theorem 4.2 and Theorem 4.3 , see the Figures $6,7,8$ and 9 .

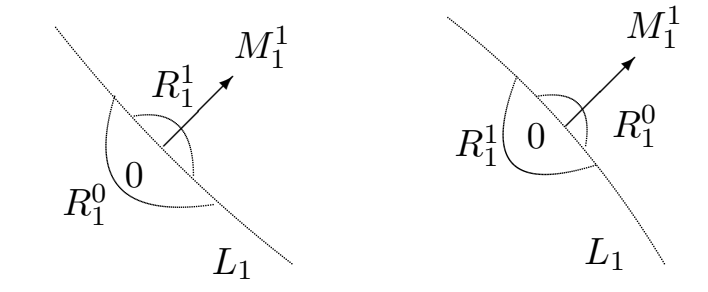

$\alpha(\mu) \geq 0, w_{1}^{12}>1 \quad \alpha(\mu) \leq 0,0<w_{1}^{12}<1$

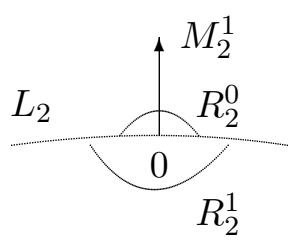

$\alpha(\mu) \geq 0, w_{2}^{12}>1$

Figure 8

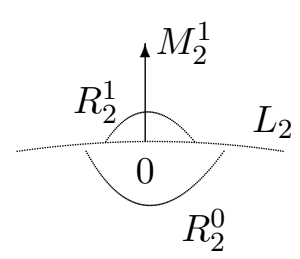

$\alpha(\mu) \leq 0,0<w_{2}^{12}<1$

Figure 6

Figure 7

Figure 9

Next we discuss the large 1-homoclinic loop and large 1-periodic orbit bifurcated by $\Gamma=\Gamma_{1} \cup \Gamma_{2}$, that is, discuss the solutions $Q\left(s_{12}, s_{21}, u_{1}^{2}, u_{2}^{2}, v_{1}^{1}, v_{2}^{1}\right)$ of the bifurcation equation (3.18) which satisfy $s_{12} \geq 0$, $s_{21} \geq 0$. By (3.15), for $0 \leq s_{12}, s_{21},|\mu| \ll 1$, the equation $\left(G_{1}^{3}, G_{1}^{4}, G_{2}^{3}, G_{2}^{4}\right)=0$ always has unique solution $u_{1}^{2}=u_{1}^{2}\left(s_{21}, s_{12}, \mu\right), u_{2}^{2}=u_{2}^{2}\left(s_{21}, s_{12}, \mu\right), v_{1}^{1}=v_{1}^{1}\left(s_{21}, s_{12}, \mu\right), v_{2}^{1}=v_{2}^{1}\left(s_{21}, s_{12}, \mu\right)$. Substituting it into $\left(G_{1}^{1}, G_{2}^{1}\right)=0$, we have

$$
\left\{\begin{array}{l}
\delta\left[-\left(w_{1}^{12}\right)^{-1} s_{21}^{(1+\alpha(\mu))}+s_{12}\right]+M_{1}^{1} \mu+\text { h.o.t. }=0, \\
\delta\left[\left(w_{2}^{12}\right)^{-1} s_{12}^{(1+\alpha(\mu))}-s_{21}\right]+M_{2}^{1} \mu+\text { h.o.t. }=0 .
\end{array}\right.
$$

Case 1. $\alpha(\mu)>0$

In this case, we have the following conclusions.

Theorem 4.4. If (H1)-(H4) are satisfied, $|\mu| \ll 1, \alpha(\mu)>0, \operatorname{rank}\left(M_{1}^{1}, M_{2}^{1}\right)=2$, then, we have the following (see Figure 10).

(1) In $\left\{M_{1}^{1} \mu<0, M_{2}^{1} \mu<0\right\}$, there exists a $(l-1)$-dimensional surface $L_{21}^{12}$ which is tangent to $L_{2}$ at $\mu=0$, and, in $\left\{M_{1}^{1} \mu>0, M_{2}^{1} \mu>0\right\}$, there exists a $(l-1)$-dimensional surface $L_{12}^{21}$ which is tangent to $L_{1}$ at $\mu=0$, such that, the necessary and sufficient condition that system (1.2) has a large homoclinic loop near $\Gamma$ is $\mu \in L_{12}^{21} \cup L_{21}^{12}$.

(2) There exists an area $R_{1}$ that bounds with $L_{12}^{21}$ and $L_{21}^{12}$, vector $M_{1}^{1}$ directs to the outside of $R_{1}$ from $L_{12}^{21}$, and $M_{2}^{1}$ directs to the inner of $R_{1}$ from $L_{21}^{12}$, such that, for $\mu \in R_{1}$, system 1.2 has a large periodic orbit near $\Gamma$.

(3) For $\mu \in L=L_{1} \cap L_{2}$, double homoclinic loops $\Gamma$ is preserved.

Where, $L_{i}, i=1,2$ are defined by $M_{i}^{1} \mu+$ h.o.t. $=0$ which are expressed by the two equations of (4.6) satisfying $s_{12}=s_{21}=0 . L_{21}^{12}$ defined by

$$
\left(w_{2}^{12}\right)^{-1}\left(-\delta^{-1} M_{1}^{1} \mu+\text { h.o.t }\right)^{(1+\alpha(\mu))}+\delta^{-1} M_{2}^{1} \mu+\text { h.o.t. }=0,
$$

and $L_{12}^{21}$ defined by

$$
\left(w_{1}^{12}\right)^{-1}\left(\delta^{-1} M_{2}^{1} \mu+\text { h.o.t. }\right)^{(1+\alpha(\mu))}-\delta^{-1} M_{1}^{1} \mu+\text { h.o.t. }=0 .
$$


Proof. By 3.15), we have $\partial\left(G_{1}^{1}, G_{2}^{1}, G_{1}^{3}, G_{2}^{3}, G_{1}^{4}, G_{2}^{4}\right) /\left.\partial Q\right|_{(Q, \mu)=0}=\operatorname{diag}(\delta,-\delta, 1,1,-1,-1)+\left(g_{i j}\right)$, where, except $g_{51}=w_{1}^{14} \delta, g_{62}=-w_{2}^{14} \delta$, other elements of $\left(g_{i j}\right)$ are all zero. So, $\left\|\partial G /\left.\partial Q\right|_{(Q, \mu)=0}\right\| \neq 0$. According to the implicit function theorem, we have, near $(Q, \mu)=(0,0)$, the equation 3.18 has a unique solution

$$
s_{21}=s_{21}(\mu), u_{1}^{2}=u_{1}^{2}(\mu), v_{1}^{1}=v_{1}^{1}(\mu), s_{12}=s_{12}(\mu), u_{2}^{2}=u_{2}^{2}(\mu), v_{2}^{1}=v_{2}^{1}(\mu),
$$

satisfies $s_{21}(0)=0, s_{12}(0)=0, u_{1}^{2}(0)=0, u_{2}^{2}(0)=0, v_{1}^{1}(0)=0, v_{2}^{1}(0)=0$.

If (4.6) has a solution $s_{12}=s_{21}=0$, then (4.6) is turned to $M_{i}^{1} \mu+$ h.o.t. $=0, i=1$, 2. So, if $\operatorname{rank}\left(M_{1}^{1}, M_{2}^{1}\right)=2$, then, when $\mu \in L=L_{1} \cap L_{2}$ and $|\mu| \ll 1$, double homoclinic loop $\Gamma$ are preserved, where, $L_{1}, L_{2}$ are expressed by the two equations of (4.6) satisfying $s_{12}=s_{21}=0$.

If 4.6 has a solution $s_{21}=0, s_{12}>0$, then 4.6 is turned to

$$
\begin{gathered}
s_{12}=-\delta^{-1} M_{1}^{1} \mu+\text { h.o.t. }=0 \\
\delta\left(w_{2}^{12}\right)^{-1}\left(-\delta^{-1} M_{1}^{1} \mu+\text { h.o.t }\right)^{(1+\alpha(\mu))}+M_{2}^{1} \mu+\text { h.o.t. }=0 .
\end{gathered}
$$

If $\operatorname{rank}\left(M_{1}^{1}, M_{2}^{1}\right)=2$, then, in $\left\{M_{1}^{1} \mu<0, M_{2}^{1} \mu<0\right\}$, 4.8) defines a $(l-1)$-dimensional surface $L_{21}^{12}$ which is tangent to $L_{2}$ at $\mu=0$, such that, system 1.2 has a unique large homoclinic loop in the neighborhood of $\Gamma$ if $\mu \in L_{21}^{12}$ and $\mu \ll 1$.

Similarly, in $\left\{M_{1}^{1} \mu>0, M_{2}^{1} \mu>0\right\}$, we can get the $(l-1)$-dimensional surface $L_{12}^{21}$ which is tangent to $L_{1}$ at $\mu=0$, such that, system 1.2 has a unique large homoclinic loop in the neighborhood of $\Gamma$ if $\mu \in L_{12}^{21}$ and $\mu \ll 1$.

If 4.6 has a solution $s_{21}>0, s_{12}>0$, then, making the derivative of (4.6) about $\mu$, we get

$$
\begin{gathered}
\left(s_{12}\right)_{\mu} M_{1}^{1}=-\delta^{-1}\left|M_{1}^{1}\right|^{2}+O(|\mu|)+O\left(s_{12}^{\alpha(\mu)}\right), \\
\left(s_{21}\right)_{\mu} M_{2}^{1}=\delta^{-1}\left|M_{2}^{1}\right|^{2}+O(|\mu|)+O\left(s_{21}^{\alpha(\mu)}\right) .
\end{gathered}
$$

The above expressions show when $|\mu| \ll 1$ and $M_{i}^{1} \neq 0, i=1,2$, in $L_{12}^{21}$, directional derivative of $s_{12}$ along $M_{1}^{1}$ is negative; in $L_{21}^{12}$, directional derivative of $s_{21}$ along $M_{2}^{1}$ is positive. Notice that $\left\{\mu: s_{12}(\mu)=\right.$ $\left.0, s_{21}(\mu)>0\right\} \subset L_{12}^{21},\left\{\mu: s_{21}(\mu)=0, s_{12}(\mu)>0\right\} \subset L_{21}^{12}$ and $\left\{\mu: s_{12}(\mu)=s_{21}(\mu)=0\right\} \subset L_{12}^{21} \cap L_{21}^{12}$, then, 4.6 has a solution satisfying $s_{12}>0, s_{21}>0$ if and only if $\mu \in R_{1}$, where, $R_{1}$ is a area which have the boundaries $L_{12}^{21}$ and $L_{21}^{12}$, and vector $M_{1}^{1}$ directs to the outside of $R_{1}$ from the boundary $L_{12}^{21}, M_{2}^{1}$ directs to the inner of $R_{1}$ from the boundary $L_{21}^{12}$. So, if $\mu \in R_{1}$ and $\mu \ll 1$, the system 1.2 has a large periodic orbit in the neighborhood of $\Gamma=\Gamma_{1} \cap \Gamma_{2}$.

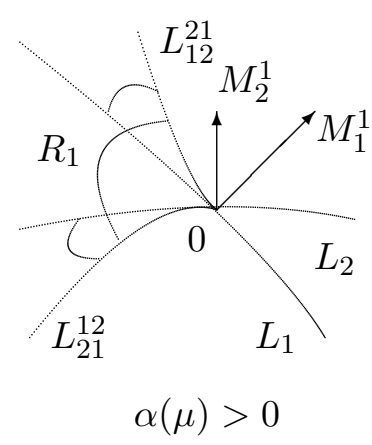

Figure 10

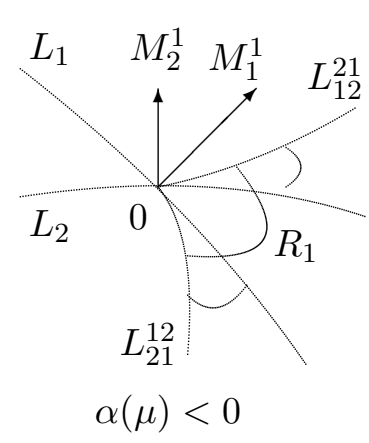

Figure 11

Case 2. $\alpha(\mu)<0$

In this case, $1+\alpha(\mu)<1$, by times scale transformations $s_{12} \rightarrow\left(s_{12}\right)^{\frac{1}{1+\alpha(\mu)}}, s_{21} \rightarrow\left(s_{21}\right)^{\frac{1}{1+\alpha(\mu)}}$, 4.6 becomes 


$$
\left\{\begin{array}{l}
-\left(w_{1}^{12}\right)^{-1} s_{21}+\left(s_{12}\right)^{\frac{1}{1+\alpha(\mu)}}+\delta^{-1} M_{1}^{1} \mu+\text { h.o.t. }=0, \\
\left(w_{2}^{12}\right)^{-1} s_{12}-\left(s_{21}\right)^{\frac{1}{1+\alpha(\mu)}}+\delta^{-1} M_{2}^{1} \mu+\text { h.o.t. }=0 .
\end{array}\right.
$$

Thus, similar to that of Theorem 4.4, we have,

Theorem 4.5. If (H1)-(H4) are satisfied, $|\mu| \ll 1, \alpha(\mu)<0, \operatorname{rank}\left(M_{1}^{1}, M_{2}^{1}\right)=2$, then, we have the following (see Figure 11).

(1) In $\left\{M_{1}^{1} \mu<0, M_{2}^{1} \mu<0\right\}$, there exists a $(l-1)$-dimensional surface $L_{21}^{12}$ which is tangent to $L_{1}$ at $\mu=0$, and, in $\left\{M_{1}^{1} \mu>0, M_{2}^{1} \mu>0\right\}$, there exists a $(l-1)$-dimensional surface $L_{12}^{21}$ which is tangent to $L_{2}$ at $\mu=0$, such that, the necessary and sufficient condition that system (1.2) has a large homoclinic loop near $\Gamma$ is $\mu \in L_{12}^{21} \cup L_{21}^{12}$.

(2) There exists an area $R_{1}$ that bounds with $L_{12}^{21}$ and $L_{21}^{12}$, vector $M_{2}^{1}$ directs to the outside of $R_{1}$ from $L_{12}^{21}$, and $M_{1}^{1}$ directs to the inner of $R_{1}$ from $L_{21}^{12}$, such that, for $\mu \in R_{1}$, system $(1.2)$ has a large periodic orbit near $\Gamma$.

(3) For $\mu \in L=L_{1} \cap L_{2}$, double homoclinic loops $\Gamma$ is preserved.

Where, $L_{i}, i=1,2$ are defined by $M_{i}^{1} \mu+$ h.o.t. $=0$ which are expressed by the two equations of 4.9) satisfying $s_{12}=s_{21}=0 . L_{21}^{12}$ defined by

and $L_{12}^{21}$ defined by

$$
\left(-\delta^{-1} w_{2}^{12} M_{2}^{1} \mu+\text { h.o.t. }\right)^{\frac{1}{1+\alpha(\mu)}}+\delta^{-1} M_{1}^{1} \mu+\text { h.o.t. }=0,
$$

$$
-\left(\delta^{-1} w_{1}^{12} M_{1}^{1} \mu+\text { h.o.t. }\right)^{\frac{1}{1+\alpha(\mu)}}+\delta^{-1} M_{2}^{1} \mu+\text { h.o.t. }=0 .
$$

Case 3. $\alpha(\mu)=0$

In this case, 4.6 becomes

$$
\left\{\begin{array}{l}
-\left(w_{1}^{12}\right)^{-1} s_{21}+s_{12}+\delta^{-1} M_{1}^{1} \mu+\text { h.o.t. }=0 \\
\left(w_{2}^{12}\right)^{-1} s_{12}-s_{21}+\delta^{-1} M_{2}^{1} \mu+\text { h.o.t. }=0 .
\end{array}\right.
$$

So,

$$
\left(\begin{array}{l}
s_{12} \\
s_{21}
\end{array}\right)=\delta^{-1} D^{-1}\left(\begin{array}{c}
\left(M_{1}^{1}-\left(w_{1}^{12}\right)^{-1} M_{2}^{1}\right) \mu \\
\left(\left(w_{2}^{12}\right)^{-1} M_{1}^{1}-M_{2}^{1}\right) \mu
\end{array}\right)+\text { h.o.t. }
$$

where, $D=\left(w_{1}^{12} w_{2}^{12}\right)^{-1}-1$.

Denote $M_{0}^{1}:=\left(w_{2}^{12}\right)^{-1} M_{1}^{1}-M_{2}^{1}, M_{0}^{2}:=M_{1}^{1}-\left(w_{1}^{12}\right)^{-1} M_{2}^{1}$. Thus, we get the following theorem.

Theorem 4.6. Suppose that (H1)-(H4) hold, $|\mu| \ll 1$. If $\alpha(\mu)=0,\left(w_{1}^{12} w_{2}^{12}\right)^{-1} \neq 1, \operatorname{rank}\left\{M_{1}^{1}, M_{2}^{1}\right\}=2$, then, 4.11) has a unique solution $0 \leq s_{12}(\mu), s_{21}(\mu) \ll 1$ satisfying $s_{12}(0)=s_{21}(0)=0$. Moreover

(1) In the region $\left\{D^{-1} M_{0}^{2} \mu>0\right\}$, there is a $(l-1)$-dimensional surface $L_{21}^{12}$ which has normal vector $M_{0}^{1}$ at $\mu=0$, such that for $\mu \in L_{21}^{12}$, (4.11) has a solution $s_{21}=0, s_{12}>0$, that is, system (1.2) has a large homoclinic loop.

(2) In the region $\left\{D^{-1} M_{0}^{1} \mu>0\right\}$, there is a $(l-1)$-dimensional surface $L_{12}^{21}$ which has normal vector $M_{0}^{2}$ at $\mu=0$, such that for $\mu \in L_{12}^{21}$, 4.11 has a solution $s_{12}=0, s_{21}>0$, that is, system (1.2) has a large homoclinic loop.

(3) If $\mu \in L_{12}^{21} \cap L_{21}^{12}$, then, 4.11 has a solution $s_{12}=0, s_{21}=0$, that is, system 1.2 has a double homoclinic loops.

(4) If $\mu \in\left\{D^{-1} M_{0}^{2} \mu>0\right\} \cap\left\{D^{-1} M_{0}^{1} \mu>0\right\}$, then, 4.11 has a solution $s_{12}>0, s_{21}>0$, that is, system (1.2) has a large periodic orbit.

Figures 12 and 13 are the bifurcation diagrams of Theorem 4.6 . 


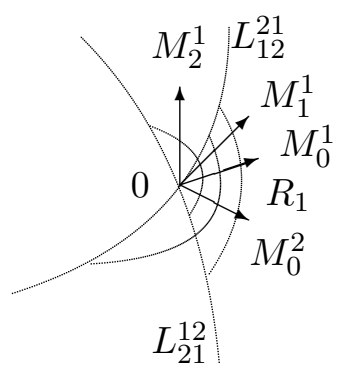

$$
\alpha(\mu)=0, D>0
$$

Figure 12

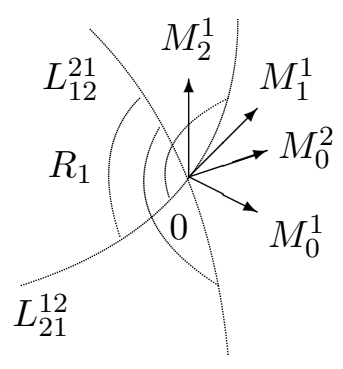

$\alpha(\mu)=0, D<0$

Figure 13

\section{Conclusion}

In this paper, we have discussed the bifurcation problems of double homoclinic loops with resonant condition for higher dimensional system. In the parameter space, for the different values of $\alpha(\mu), w_{1}^{12}$, and $w_{2}^{12}$, we obtain the existence, number and existence regions of the small homoclinic loops, periodic orbits, and large homoclinic loops, periodic orbits, respectively.

Finally, combining the related results of Theorems 4.1 4.6, we can get the complete bifurcations figures in the parameter space for the different values of $\alpha(\mu), w_{1}^{12}$, and $w_{2}^{12}$.

Theorem 5.1. Suppose that $\mathbf{( H 1 )}-(\mathbf{H} 4)$ hold, $|\mu| \ll 1, \operatorname{rank}\left\{M_{1}^{1}, M_{2}^{1}\right\}=2$, then, we have the following conclusions.

(1) For the case $\alpha(\mu)>0,0<w_{1}^{12}<1,0<w_{2}^{12}<1$, the bifurcations figure is the combination of Figures 2, 4 and 10 .

(2) For the case $\alpha(\mu)<0, w_{1}^{12}>1, w_{2}^{12}>1$, the bifurcations figure is the combination of Figures 3 , 5 and 11.

(3) For the case $\alpha(\mu)>0,0<w_{1}^{12}<1, w_{2}^{12}>1$, the bifurcations figure is the combination of Figures 2 , 8 and 10.

(4) For the case $\alpha(\mu)>0, w_{1}^{12}>1,0<w_{2}^{12}<1$, the bifurcations figure is the combination of Figures 6 , 4 and 10.

(5) For the case $\alpha(\mu)>0, w_{1}^{12}>1, w_{2}^{12}>1$, the bifurcations figure is the combination of Figures 6, 8 and 10.

(6) For the case $\alpha(\mu)<0, w_{1}^{12}>1,0<w_{2}^{12}<1$, the bifurcations figure is the combination of Figures 3 , 9 and 11 .

(7) For the case $\alpha(\mu)<0,0<w_{1}^{12}<1, w_{2}^{12}>1$, the bifurcations figure is the combination of Figures 7 , 5 and 11.

(8) For the case $\alpha(\mu)<0,0<w_{1}^{12}<1,0<w_{2}^{12}<1$, the bifurcations figure is the combination of Figures 7. 9 and 11 .

(9) For the case $\alpha(\mu)=0, w_{1}^{12}>1, w_{2}^{12}>1, D=\left(w_{1}^{12} w_{2}^{12}\right)^{-1}-1<0$, the bifurcations figure is the combination of Figures 6, 8 and 13 .

(10) For the case $\alpha(\mu)=0,0<w_{1}^{12}<1,0<w_{2}^{12}<1, D=\left(w_{1}^{12} w_{2}^{12}\right)^{-1}-1>0$, the bifurcations figure is the combination of Figures 7,9 and 12 . 
(11) For the case $\alpha(\mu)=0, w_{1}^{12}>1,0<w_{2}^{12}<1, D=\left(w_{1}^{12} w_{2}^{12}\right)^{-1}-1>0$, the bifurcations figure is the combination of Figures 6,9 and 12 .

(12) For the case $\alpha(\mu)=0, w_{1}^{12}>1,0<w_{2}^{12}<1, D=\left(w_{1}^{12} w_{2}^{12}\right)^{-1}-1<0$, the bifurcations figure is the combination of Figures 6, 9 and 13.

(13) For the case $\alpha(\mu)=0,0<w_{1}^{12}<1, w_{2}^{12}>1, D=\left(w_{1}^{12} w_{2}^{12}\right)^{-1}-1>0$, the bifurcations figure is the combination of Figures 7, 8 and 12 .

(14) For the case $\alpha(\mu)=0,0<w_{1}^{12}<1, w_{2}^{12}>1, D=\left(w_{1}^{12} w_{2}^{12}\right)^{-1}-1<0$, the bifurcations figure is the combination of Figures 7,8 and 13 .

For example, the bifurcations figure for the case (1) $\left(\alpha(\mu)>0,0<w_{1}^{12}<1,0<w_{2}^{12}<1\right)$ is the following Figure 14, the bifurcations figure for the case (2) $\left(\alpha(\mu)<0, w_{1}^{12}>1, w_{2}^{12}>1\right)$ is the following Figure 15. Here, the relative positions of $\Sigma_{1}, \Sigma_{2}$ and $L_{12}^{21}, L_{21}^{12}$ are determined by their expressions as follows:

(i) For the case $\alpha(\mu)>0,0<w_{1}^{12}<1,0<w_{2}^{12}<1$,

$$
\left.M_{1}^{1} \mu\right|_{\mu \in L_{12}^{21}}>\left.M_{1}^{1} \mu\right|_{\mu \in \Sigma_{1}}>0,\left.\quad M_{2}^{1} \mu\right|_{\mu \in L_{21}^{12}}<\left.M_{2}^{1} \mu\right|_{\mu \in \Sigma_{2}}<0 .
$$

(ii) For the case $\alpha(\mu)<0, w_{1}^{12}>1, w_{2}^{12}>1$,

$$
\left.M_{1}^{1} \mu\right|_{\mu \in L_{21}^{12}}<\left.M_{1}^{1} \mu\right|_{\mu \in \Sigma_{1}}<0,\left.\quad M_{2}^{1} \mu\right|_{\mu \in L_{12}^{21}}>\left.M_{2}^{1} \mu\right|_{\mu \in \Sigma_{2}}>0 .
$$
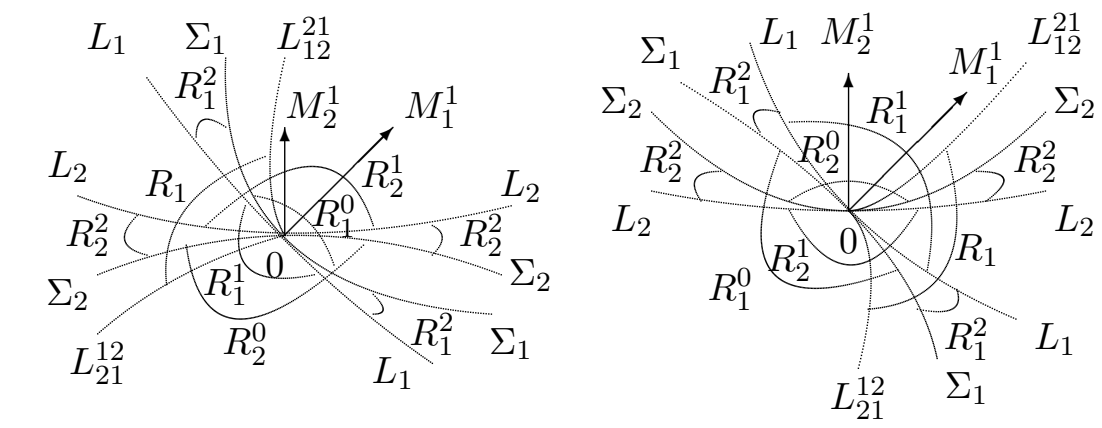

$$
\alpha(\mu)>0,0<w_{1}^{12}<1,0<w_{2}^{12}<1
$$

$\alpha(\mu)<0, w_{1}^{12}>1, w_{2}^{12}>1$

Figure 14

Figure 15

\section{Acknowledgments}

This work is supported by the National Natural Science Foundation of China (No. 10671069), Shandong Province Natural Science Foundation (ZR2015AL005) and the Applied Mathematics Enhancement Program of Linyi University.

\section{References}

[1] S.-N. Chow, B. Deng, B. Fiedler, Homoclinic bifurcation at resonant eigenvalues, J. Dynam. Differential Equations, 2 (1990), 177-244. 1

[2] M. Han, Y. Wu, The stability of double homoclinic loops, Appl. Math. Lett., 17 (2004), 1291-1298. 1

[3] Y. Jin, F. Li, H. Xu, J. Li, L. Zhang, B. Ding, Bifurcations and stability of nondegenerated homoclinic loops for higher dimensional systems, Comput. Math. Methods Med., 2013 (2013), 9 pages. 1, 2 , 4

[4] Y. Jin, D. Zhu, Degenerated homoclinic bifurcations with higher dimensions, Chinese Ann. Math. Ser., 21 (2000), 201-210. 1, 2 
[5] Y. L. Jin, D. M. Zhu, Twisted bifurcations and stability of homoclinic loop with higher dimensions, Appl. Math. Mech., 25 (2004), 1176-1183. 1, 2

[6] X. Liu, D. Zhu, On the stability of homoclinic loops with higher dimension, Discrete Contin. Dyn. Syst. Ser., 17 (2012), 915-932. 1. 2

[7] Q. Lu, Codimension 2 bifurcation of twisted double homoclinic loops, Comput. Math. Appl., 57 (2009), $1127-1141$. 1., 2

[8] D. Luo, M. Han, D. Zhu, The uniqueness of limit cycles bifurcating from a singular closed orbit (I), Acta Math. Sinica, 35 (1992), 407-417. 1

[9] K. J. Palmer, Exponential dichotomies and transversal homoclinic points, J. Differential Equations, 55 (1984), 225-256. 2

[10] C. G. Ragazzo, On the Stability of Double Homoclinic Loops, Comm. Math. Phys., 184 (1997), 251-272. 1

[11] S. Wiggins, Introduction to Applied Nonlinear Dynamical Systems and Chaos: Second Edition, Springer-Verlag, New York, (2003). 1

[12] W. Zhang, D. Zhu, Codimension 2 bifurcations of double homoclinic loops, Chaos Solitons Fractals, 39 (2009), 295-303. 11, 2

[13] D. Zhu, Problems in homoclinic bifurcation with higher dimensions, Acta Math. Sinica, 14 (1998), 341-352. 1, 2 